\title{
Altered brain function, structure, and developmental trajectory in children born late preterm
}

\author{
Jane E. Brumbaugh', Amy L. Conrad', Jessica K. Lee², lan J. DeVolder², M. Bridget Zimmerman², Vincent A. Magnotta ${ }^{4}$, \\ Eric D. Axelson ${ }^{2}$ and Peggy C. Nopoulos ${ }^{1,2,5}$
}

BACKGROUND: Late preterm birth (34-36wk gestation) is a common occurrence with potential for altered brain development.

METHODS: This observational cohort study compared children at age 6-13 y based on the presence or absence of the historical risk factor of late preterm birth. Children completed a battery of cognitive assessments and underwent magnetic resonance imaging of the brain.

RESULTS: Late preterm children $(n=52)$ demonstrated slower processing speed $(P=0.035)$ and scored more poorly in visualspatial perception $(P=0.032)$ and memory $(P=0.007)$ than full-term children $(n=74)$. Parents of late preterm children reported more behavioral difficulty $(P=0.004)$. There were no group differences in cognitive ability or academic achievement. Imaging revealed similar intracranial volumes but less total tissue and more cerebrospinal fluid $(P=0.004)$ for late preterm children compared to full-term children. The tissue difference was driven by differences in the cerebrum $(P=$ $0.028)$ and distributed across cortical $(P=0.051)$ and subcortical tissue $(P=0.047)$. Late preterm children had a relatively smaller thalamus $(P=0.012)$ than full-term children. Only fullterm children demonstrated significant decreases in cortical tissue volume $(P<0.001)$ and thickness $(P<0.001)$ with age. CONCLUSION: Late preterm birth may affect cognition, behavior, and brain structure well beyond infancy.

ate preterm (PT) birth (34-36 wk gestation) accounts for $8 \%$ -of births in the United States each year, making late preterm birth a public health issue $(1,2)$. Because a significant portion of brain growth occurs in the final weeks of gestation, late PT children may be susceptible to altered brain development (3). There is an increased incidence of school suspension, grade retention, and special education among late PT children compared to full-term (FT) children (37-41 wk gestation) (4-6). The neuroanatomical underpinnings for this discrepant early achievement in late PT children are not yet fully understood.

Magnetic resonance imaging (MRI) is one tool to understand what brain structures and functions show altered developmental patterns in children born PT. A meta-analysis of 15 brain imaging studies reflective of 818 very PT ( $<32 \mathrm{wk}$ gestation) children found smaller total brain volumes with reduced gray and white matter volumes compared to FT children (7). Looking at children spared perinatal neurological injury, Lax et al. (8) found decreased brain volume, cortical surface area, and cortical thickness as well as smaller basal ganglia and thalamic volumes in very PT children. Limited imaging studies focused on late PT infants have demonstrated smaller biparietal diameter, larger extra-axial spaces, and smaller gray matter volume at term corrected age compared to FT infants $(9,10)$. Beyond the neonatal period, Rogers et al. (11) found less total gray matter as well as smaller right temporal and parietal lobes in late PT children at school age.

The developmental trajectory of brain structures, in particular the cerebral cortex, may be more relevant to brain function, or cognition, than absolute size. The cortex has an inverted U-shaped trajectory in the typically developing brain across childhood and adolescence (12). Reductions in the cortex are related to maturation, including synaptic pruning and myelination at the gray-white matter border (13). The trajectory of cortical thickness across age is more predictive of intelligence among typically developing children than absolute cortical thickness, supporting the importance of evaluating trajectories (14). Ment et al. (15) performed serial MRI in very PT children at 8-12 y of age and showed less gray matter reduction with less white matter gain over time compared to FT children.

To better understand what underlies the discrepant functional outcomes of late PT children, we performed a cohort study to evaluate brain function, structure, and cortical trajectories at school age using a multi-modal approach. We hypothesized late PT children would have subtle differences in cognition, behavior, and brain structure compared to their FT peers.

\section{RESULTS}

\section{Growth}

By design, late PT children had a lower birth weight than FT children $(P<0.001)$ (Table 1$)$. At testing, PT children were

\footnotetext{
'Stead Family Department of Pediatrics, University of lowa, lowa City, lowa; ${ }^{2}$ Department of Psychiatry, University of lowa, lowa City, lowa; ${ }^{3}$ Department of Biostatistics, University of lowa, lowa City, lowa; ${ }^{4}$ Department of Radiology, University of lowa, lowa City, lowa; ${ }^{5}$ Department of Neurology, University of lowa, lowa City, lowa. Correspondence: Jane E. Brumbaugh (jane-brumbaugh@uiowa.edu)

Received 17 December 2015; accepted 29 February 2016; advance online publication 11 May 2016. doi:10.1038/pr.2016.82
} 


\section{Articles | Brumbaugh et al.}

significantly shorter $(P<0.001)$ and lighter $(P=0.024)$. There was no significant difference in BMI $\mathrm{z}$-score $(P=0.332)$ or head circumference $(P=0.112)$ between PT and FT children. There were significant group by sex interactions for weight $\mathrm{z}$-score $(P=0.036)$ and BMI z-score $(P=0.030)$; differences were observed between PT and FT boys that were not observed between PT and FT girls. The mean weight $\mathrm{z}$-score was less in PT boys compared to FT boys $(P=0.002)$ while there was no difference between PT and FT girls $(P=0.903)$. PT boys also had a lower mean BMI z-score than FT boys $(P=0.034)$ while

Table 1. Demographics and anthropometrics

\begin{tabular}{|c|c|c|c|c|c|c|}
\hline & \multicolumn{2}{|c|}{ Full term $(n=74)$} & \multicolumn{2}{|c|}{ Late preterm $(n=52)$} & \multirow{2}{*}{$\begin{array}{c}\text { Mean difference } \\
95 \% \mathrm{Cl}^{\mathrm{a}}\end{array}$} & \multirow[b]{2}{*}{$P$} \\
\hline & Mean & $95 \% \mathrm{Cl}$ & Mean & $95 \% \mathrm{Cl}$ & & \\
\hline \multicolumn{7}{|l|}{ Demographics } \\
\hline Birth weight ${ }^{b}(\mathrm{~kg})$ & 3.59 & $3.48,3.70$ & 2.70 & $2.57,2.83$ & $0.72,1.05$ & $<0.001$ \\
\hline $\operatorname{Age}^{b}(y)$ & 10.00 & $9.52,10.48$ & 9.44 & $8.87,10.01$ & $-0.20,1.30$ & 0.146 \\
\hline SESc & 2.26 & $2.14,2.38$ & 2.30 & $2.16,2.45$ & $-0.23,0.15$ & 0.647 \\
\hline Male sex ${ }^{d}(\%)$ & $37(50.0)$ & - & $29(55.8)$ & - & - & 0.523 \\
\hline Caucasian, non-Hispanic ${ }^{d}(\%)$ & $62(83.8)$ & - & $45(88.2)$ & - & - & 0.486 \\
\hline \multicolumn{7}{|l|}{ Anthropometrics } \\
\hline Height (cm) & 141.76 & $140.36,143.15$ & 137.77 & $136.10,139.44$ & $1.80,6.17$ & $<0.001$ \\
\hline Height z-score & 0.64 & $0.43,0.85$ & -0.06 & $-0.32,0.20$ & $0.34,1.00$ & $<0.001$ \\
\hline Weight (kg) & 37.64 & $35.47,39.81$ & 36.08 & $33.48,38.67$ & $-1.84,4.97$ & 0.364 \\
\hline Weight z-score & 0.57 & $0.34,0.80$ & 0.16 & $-0.12,0.43$ & $0.06,0.78$ & 0.024 \\
\hline $\mathrm{BMI}^{\mathrm{e}}\left(\mathrm{kg} / \mathrm{m}^{2}\right)$ & 18.25 & $17.42,19.07$ & 18.36 & $17.37,19.35$ & $-1.41,1.18$ & 0.860 \\
\hline BMI z-score & 0.39 & $0.16,0.62$ & 0.21 & $-0.06,0.49$ & $-0.19,0.54$ & 0.332 \\
\hline $\operatorname{OFC}^{f}(\mathrm{~cm})$ & 54.59 & $54.02,55.15$ & 53.87 & $53.19,54.55$ & $-0.17,1.60$ & 0.112 \\
\hline
\end{tabular}

Late preterm children were shorter and lighter than full-term children at school age.

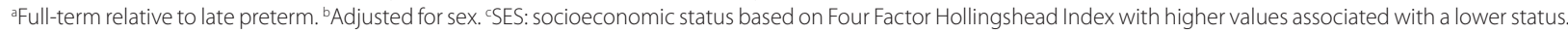

${ }^{d}$ Number (percentage) reported for dichotomous variables. ${ }^{\circledR B M I}$, body mass index. ${ }^{\dagger} \mathrm{OFC}$, orbitofrontal circumference

Table 2. Functional domains

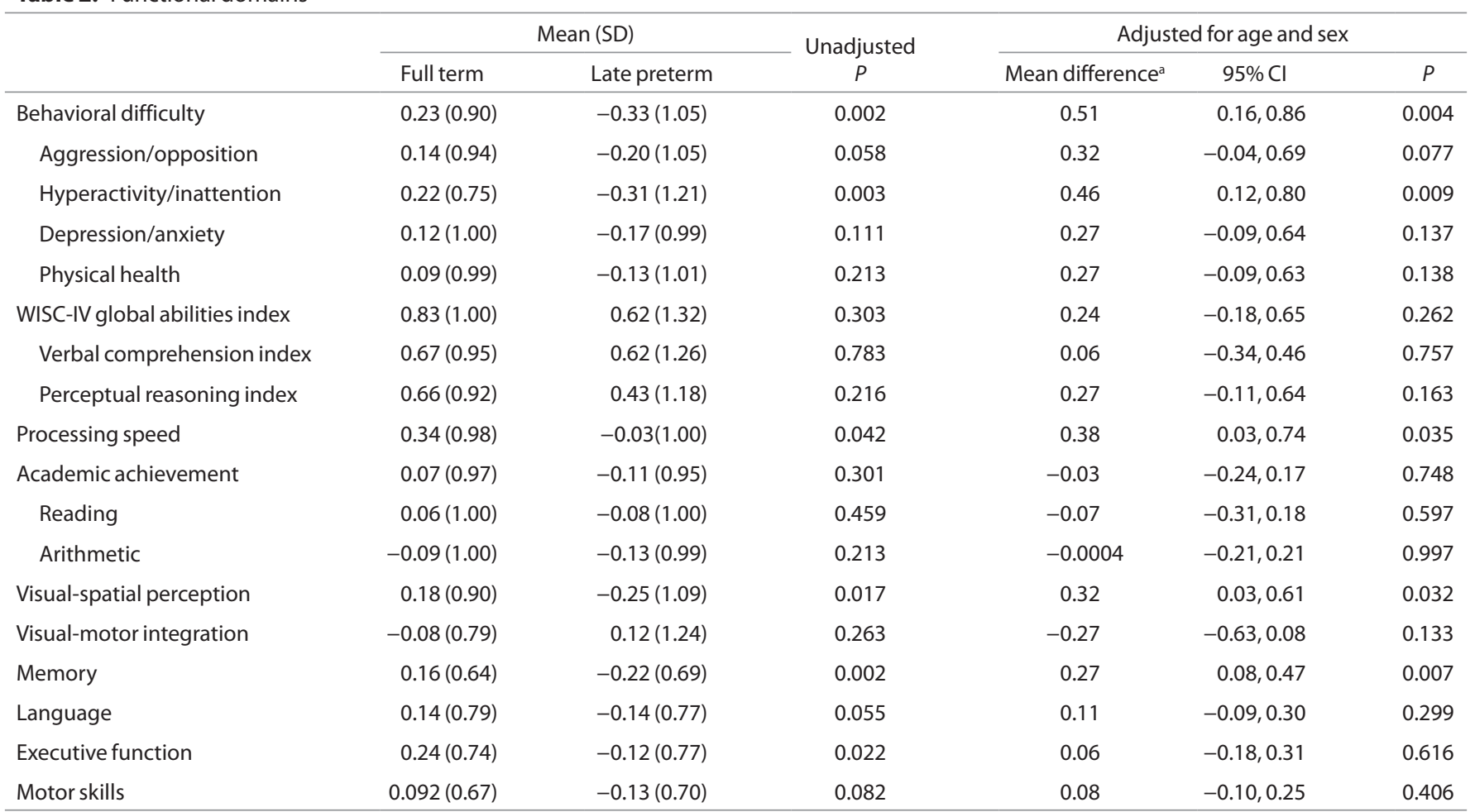

Late preterm children had more difficulties in behavior, processing speed, visual-spatial perception, and memory. Reported values are $z$-scores where higher values reflect better functioning.

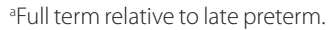


there was no difference between PT and FT girls $(P=0.419)$. There were no significant ages by group interactions on the anthropometric measurements.

\section{Behavior, Cognition, and Motor Skills}

Parents of late PT children reported more behavioral problems $(P=0.004)$ (Table 2$)$. The group difference in the behavior domain was driven by the externalizing behaviors of hyperactivity/inattention $(P=0.009)$ and aggression/opposition $(P=$ 0.077 ). There were no significant ages by group or sex by group interactions on the behavioral difficulty domain.

Late PT children had similar cognitive ability as FT children $(P=0.262)$ with no significant group difference when broken down into verbal comprehension $(P=0.757)$ and perceptual reasoning $(P=0.163)$. Late $\mathrm{PT}$ children demonstrated slower processing speed than FT children $(P=0.035)$. Functionally, there were no differences between PT and FT children on academic achievement measures of reading $(P=0.597)$ or arithmetic $(P=0.997)$. There was no significant age by group or sex by group interactions for the cognitive ability, processing speed, or academic achievement domains.
PT children performed more poorly than FT children in two neuropsychological domains: visual-spatial perception $(P=0.032)$ and memory $(P=0.007)$ (Table 2$)$. There were no significant group differences in the visual-motor integration $(P=0.133)$, language $(P=0.299)$, or executive function $(P=0.616)$ domains. There were no significant ages by group or sex by group interactions on the neuropsychological domains.

Motor skills assessment revealed no group difference between PT and FT children $(P=0.406)$. There were no significant ages by group or sex by group interactions on the motor domain.

\section{Brain Structure}

The late PT cohort had a similar intracranial volume (ICV) as the FT cohort $(P=0.306)$ (Table 3$)$. However, the late PT cohort had less total tissue $(P=0.004)$ and more cerebrospinal fluid $(P=0.004)$ contained within the ICV. The low total tissue volume was not tissue-specific to white or gray matter. Total white matter $(P=0.182)$ and total gray matter $(P=0.310)$ were descriptively lower in the PT group. The difference in total tissue was driven by a difference in the cerebrum $(P=0.028)$ that was distributed between both the cortex $(P=0.051)$ and

Table 3. Structural measures

\begin{tabular}{|c|c|c|c|c|c|c|}
\hline & \multicolumn{2}{|c|}{ Full term $(n=72)^{\mathrm{a}}$} & \multicolumn{2}{|c|}{ Preterm $(n=48)$} & \multirow{2}{*}{$\begin{array}{c}\text { Mean difference } \\
95 \% \mathrm{Cl}^{c}\end{array}$} & \multirow[b]{2}{*}{$P$} \\
\hline & Mean $^{b}$ & $95 \% \mathrm{Cl}$ & Mean ${ }^{b}$ & $95 \% \mathrm{Cl}$ & & \\
\hline$I C V^{d}$ & $1,383.143$ & $1,352.503,1,413.784$ & $1,409.225$ & $1,371.238,1,447.211$ & $-76.288,24.124$ & 0.306 \\
\hline Tissue & 0.964 & $0.961,0.967$ & 0.956 & $0.952,0.960$ & $0.003,0.013$ & 0.004 \\
\hline White matter & 0.328 & $0.324,0.333$ & 0.324 & $0.318,0.329$ & $-0.002,0.012$ & 0.182 \\
\hline CSF & 0.036 & $0.033,0.039$ & 0.044 & $0.040,0.048$ & $-0.013,-0.003$ & 0.004 \\
\hline \multicolumn{7}{|l|}{ Tissue measures } \\
\hline Cerebrum & 0.847 & $0.844,0.851$ & 0.841 & $0.837,0.845$ & $0.001,0.012$ & 0.028 \\
\hline Cerebellum & 0.103 & $0.101,0.105$ & 0.103 & $0.101,0.105$ & $-0.002,0.003$ & 0.702 \\
\hline Brainstem $^{f}$ & 2.422 & $2.362,2.482$ & 2.351 & $2.278,2.424$ & $-0.023,0.166$ & 0.137 \\
\hline \multicolumn{7}{|l|}{ Cortical gray measures } \\
\hline Volume ${ }^{g}$ & 422.979 & $418.931,427.027$ & 418.039 & $413.022,423.055$ & $-1.523,11.405$ & 0.133 \\
\hline Surface area ${ }^{g}$ & 124.834 & $123.833,125.834$ & 123.124 & $121.885,124.364$ & $0.112,3.307$ & 0.036 \\
\hline Thickness & 2.927 & $2.905,2.949$ & 2.932 & $2.904,2.959$ & $-0.040,0.031$ & 0.789 \\
\hline \multicolumn{7}{|l|}{ Subcortical structures ${ }^{f}$} \\
\hline Hippocampus & 0.265 & $0.257,0.272$ & 0.259 & $0.250,0.269$ & $-0.006,0.018$ & 0.348 \\
\hline Thalamus & 0.924 & $0.908,0.941$ & 0.890 & $0.870,0.911$ & $0.008,0.060$ & 0.012 \\
\hline Caudate & 0.537 & $0.522,0.553$ & 0.522 & $0.503,0.540$ & $-0.009,0.040$ & 0.205 \\
\hline
\end{tabular}

Late preterm children had less tissue, more cerebrospinal fluid, and smaller thalami than full-term children.

a Mean $(95 \% \mathrm{Cl})$ for brain structural measures. ${ }^{b}$ Adjusted for age and sex ${ }^{\mathrm{C}}$ Full term relative to late preterm. ${ }^{\mathrm{d}} \mathrm{CV}$ analysis controlled for height. ${ }^{\mathrm{V}} \mathrm{Columes}$ reported as ratios to ICV except

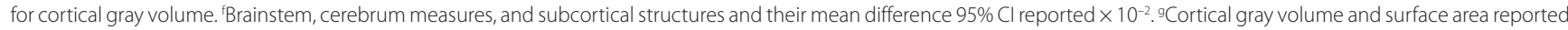
as ratios to total tissue.

CSF, cerebrospinal fluid; $\mathrm{Cl}$, confidence interval; ICV, intracranial volume. 


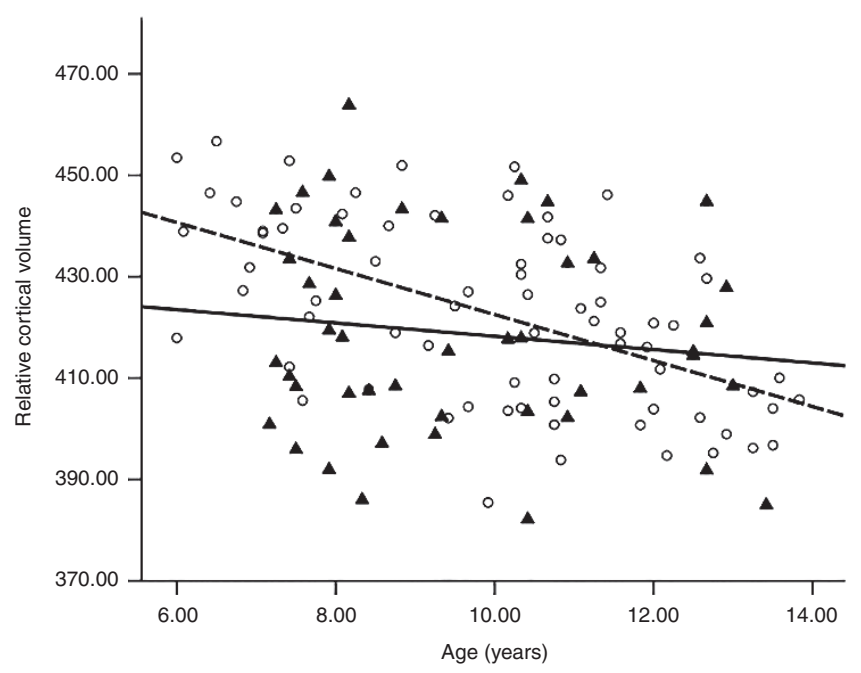

Figure 1. Developmental trajectory of cortical volume. Relative cortical tissue volume decreased with age in full-term children more than in late preterm children $(z=-2.34, P=0.019)$. Open circles and dashed line represent full-term children. Filled triangles and solid line represent late preterm children.

subcortex $(P=0.047)$; PT children had smaller cortical and subcortical tissue volumes. There was no difference in the relative cerebellar $(P=0.702)$ or brainstem volume $(P=0.137)$ between groups.

For cortical gray matter, there were three measures of interest: tissue volume, surface area, and thickness. The relative cortical tissue volume was not statistically different for FT and late PT children $(P=0.133)$, although there was less relative cortical surface area for late PT children $(P=0.036)$ than FT children. There was no significant difference in the cortical thickness between the two groups $(P=0.789)$.

While there was no main effect of group on cortical tissue volume or thickness, both the relative cortical volume $\left(R^{2} \Delta=\right.$ 0.029 , standardized $\beta=0.210,95 \%$ confidence interval (CI): $0.04,6.24 ; P=0.047)$ and cortical thickness $\left(R^{2} \Delta=0.027\right.$, standardized $\beta=0.204,95 \%$ CI: $-0.001,0.034 ; P=0.060$ ) demonstrated an effect of age that varied by group. Among FT children, the relative cortical tissue volume decreased with age $\left(\beta_{\mathrm{FT}}=-0.509,95 \% \mathrm{CI}:-5.89,-2.49 ; P<0.001\right)$ (Figure 1). There was no such effect of age on the relative cortical volume among late PT children $\left(\beta_{\mathrm{PT}}=-0.113,95 \%\right.$ CI: $-3.93,1.72 ; P=0.436)$. Cortical thickness also decreased with age among FT children $\left(\beta_{\mathrm{FT}}=-0.468,95 \% \mathrm{CI}:-0.03\right.$, $-0.01 ; P<0.001)$ while no such effect of age on cortical thickness was observed among late PT children $\left(\beta_{\mathrm{PT}}=-0.069,95 \%\right.$ CI: $-0.02,0.01 ; P=0.634$ ) (Figure 2).

Within the cerebrum, there was no main effect of group on relative white matter volume (95\% CI: $-0.003,0.010$; $P=0.272)$ and no interaction between age and group on cerebral white matter volume $\left(R^{2} \Delta=0.008\right.$, standardized $\beta=$ $-0.110,95 \%$ CI: $-0.005,0.001 ; P=0.273)$. Both PT and FT children showed gains in cerebral white matter over time $\left(\beta_{\mathrm{FT}}\right.$ $=0.548,95 \%$ CI: 0.003, 0.007; $P<0.001 ; \beta_{\mathrm{PT}}=0.322,95 \%$ CI: $0.0003,0.0061 ; P=0.027)$.

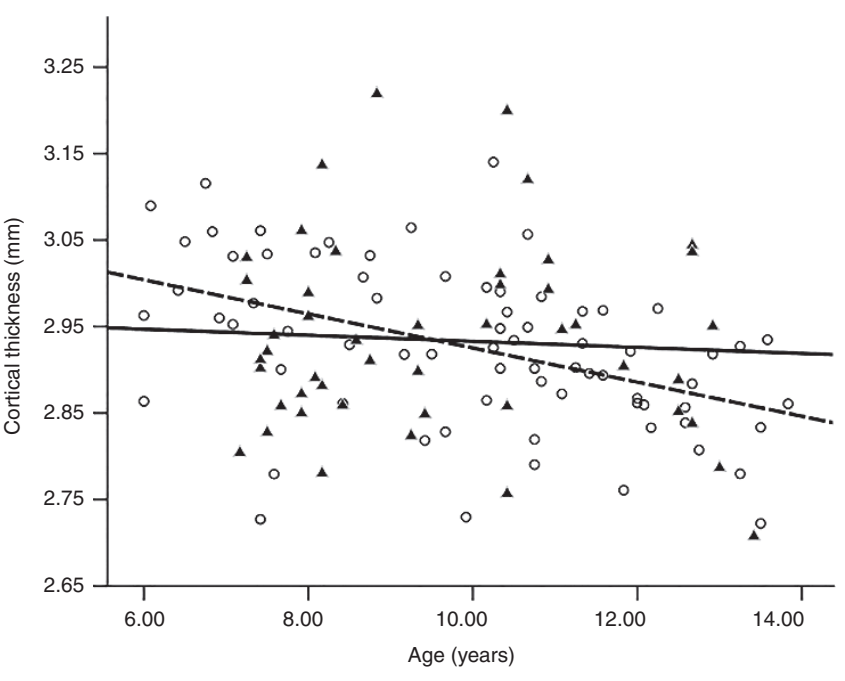

Figure 2. Developmental trajectory of cortical thickness. Cortical thickness decreased with age in full-term children more than in late preterm children $(z=-2.29, P=0.022)$. Open circles and dashed line represent full-term children. Filled triangles and solid line represent late preterm children.

For subcortical structures, PT children had a relatively smaller thalamus than FT children $(P=0.012)$. Other subcortical structures relative to ICV did not differ between PT and FT children. There were no ages by group interactions affecting subcortical structures. There were no sexes by group interactions affecting any brain structural measures.

\section{DISCUSSION}

This study provides a broad perspective on brain development following late PT birth with functional and structural measures. Functionally, parents of late PT children reported more behavioral difficulties. Late PT children demonstrated more difficulty with processing speed, visual-spatial perception, and memory compared to FT children. In contrast, late PT and FT children demonstrated similar performance in language, executive function, and motor skills. Structurally, PT children had less total tissue and more cerebrospinal fluid (CSF) than FT children. PT children did not demonstrate the effects of age on cortical tissue volume or cortical thickness that were observed in FT children. Late PT birth affects brain function and structure well beyond infancy, an impact that may not be apparent on global measures of cognitive ability.

This study brings attention to long-term growth for late PT children. The late PT cohort was shorter and lighter at school age than the FT cohort. Historically, late PT birth has not been considered a risk factor for altered growth in childhood. Recent data from Japan found late PT children to be twofold more likely to have short stature at $3 \mathrm{y}$ of age compared to FT children (16). Despite greater differences in height than weight, the late PT and FT cohorts had similar mean BMI, a relevant negative finding in an era of childhood obesity. Linear growth discrepancies are clinically significant with a relationship between linear growth and neurodevelopmental outcome demonstrated in the very-low-birth-weight preterm population (17). 
In terms of behavior, parents provided insights into behavior that may contribute to discrepant outcomes for late PT and FT children. There is evidence from larger populations that attention deficit hyperactivity disorder affects late PT children more often than FT children. In a Swedish cohort study, late PT children had 30-40\% greater likelihood of attention deficit hyperactivity disorder medication use compared to FT children (18).

For cognition, late PT children had more difficulty on measures of processing speed and memory. This is consistent with the literature on very PT children in whom discrepant processing speed and working memory relative to FT peers account for differences in academic achievement and need for special education (19). For this sample, there was no difference in academic achievement as assessed by a decoding measure and a computation measure. However, untimed academic achievement measures may not reflect functional or clinical significance, particularly when late PT children demonstrated a slower processing speed. Late PT children had more difficulties on visual-spatial perception and visual memory tasks than on language or verbal tasks, a pattern observed in very PT cohorts as well (20). In contrast, Baron et al. (21) found that late PT children had more difficulty on tasks of visual-motor integration and had difficulty with verbal fluency at $3 \mathrm{y}$ of age compared to FT children.

MRI demonstrated the presence of more CSF in the late PT brain at school age. This previously was reported at term corrected age using more crude assessment of CSF (10). The lack of tissue specificity in the tissue decrement among late PT children suggests that there may be a global effect impacting gray and white matter alike. Shifts in tissue composition were less pronounced in the late PT cohort compared to the FT cohort with less gray matter loss and less white matter gain among late PT children across the age span.

In contrast to previous work, we found a difference in cortical surface area but not in cortical thickness for late PT relative to FT children (11). We built upon the work by Rogers et al. by analyzing the developmental trajectory of cortex with age. FT children demonstrated cortical thinning with age while late PT children lacked cortical thinning across the same age span. Late PT children may have more rapid pruning at a younger age. Alternatively, PT children may have delayed or absent pruning resulting in a lack of cortical thinning.

Lastly, the thalamus, a brain region involved in sensory and motor signaling, was relatively smaller in the PT cohort compared to the FT cohort after controlling for total tissue volume. This is consistent with the pattern observed in very PT samples compared to full-term children $(22,23)$.

Strengths of this study include building on the limited literature on brain structure in the late PT population at school age (11). The brain structural data provided preliminary evidence for structural differences in the late PT brain that may underlie differences in behavior and cognition. Second, the late PT cohort came from an enriched background suggesting that the differences between PT and FT children were related to prematurity rather than a disadvantaged social situation associated with prematurity (24). Third, all children were scanned on the same scanner, thereby avoiding the introduction of tissue volume variation with multiple scanners. Finally, the breadth of assessment tools used provided a comprehensive profile of behavior, cognition, and motor skills.

Limitations of this study included the relatively small sample size, ambidirectional rather than longitudinal design, and retrospective collection of birth history data. There were multiple examiners with no formal inter-rater reliability testing performed. A licensed psychologist oversaw training of examiners with a period of direct observation followed by mentored administration of tests prior to independent administration. Additionally, the brain structural measures that differed between groups were relatively global (e.g., total tissue volume). Meanwhile, the cognitive findings that differed were relatively specific (e.g., visual-spatial perception). This made it challenging to demonstrate direct structure-function relationships.

In summary, this cohort of late PT children demonstrated more difficulty with processing speed, visual-spatial perception, and memory. Late PT children had less total brain tissue, more cerebrospinal fluid, and smaller thalami compared to FT children. Together, the behavioral, cognitive, and structural findings suggest that late PT birth should be considered a potential insult on the developing brain given the differences persisting at school age.

\section{METHODS}

We identified late PT children born between 2000 and 2006 through the University of Iowa Neonatal Admissions Registry. Children were recruited at 6 to $13 \mathrm{y}$ of age by letter. If parents expressed interest, a telephone screen was conducted to confirm the child's eligibility. We compared late PT children to healthy, typically developing FT children born between 1996 and 2006 who were recruited independently for another study involving the same behavioral and cognitive assessments and using the same MRI machine and acquisition sequences. Parents of FT children consented to share data for additional investigations on brain development. Exclusion criteria included multiple birth, major medical disease, neurological injury, 5-min Apgar score $<7$, neonatal sepsis, and birth weight $<1,500 \mathrm{~g}$ for late PT children and $<, 2500 \mathrm{~g}$ for FT children.

Written consent was obtained from a parent. Children aged 8-13 y provided written assent. Six- and 7-y-old children provided verbal assent. Children and parents were compensated for their participation. The study was approved by the University of Iowa Institutional Review Board.

There were 52 children in the late PT cohort and 74 children in the FT cohort for anthropometric, behavioral, cognition, and motor skills assessment (Table 1). Comparison of demographic variables between groups showed no difference in sex distribution $(P=0.523)$, mean age at participation $(P=0.146)$, or socioeconomic status using a modified Hollingshead Four-Factor Index of Socioeconomic Status $(P=0.647)$ (25). The modified index includes parent occupation, sex, marital status, and one Likert scale question pertaining to household wealth and education. Higher values indicate lower socioeconomic status on this scale. For imaging analysis, 48 late PT and 72 FT children successfully completed the MRI scan. Children with incomplete MRI data had motion artifact (three late PT children) or had the scan sequences stopped early or not attempted (one late PT, two FT children).

\section{Outcome Measures}

Parents completed the Pediatric Behavior Scale-30 (PBS) (26). The behavioral difficulty domain was calculated from z-scores for the PBS. On the $\mathrm{z}$-score scale, the mean is 0.00 and the $\mathrm{SD}$ is 1.00 . Scores ranging from -0.99 to 0.99 are within the average range. Children's cognitive 
ability was assessed with four subtests of the Wechsler Intelligence Scale for Children (WISC-IV): Block Design, Matrix Reasoning, Vocabulary, and Similarities (27). Z-scores for the General Ability Index, Verbal Comprehension Index, and Perceptual Reasoning Index were calculated. The processing speed domain was calculated from z-scores for the WISC-IV Processing Speed Index. Academic achievement in reading and mathematics was measured with the Wide Range Achievement Test (28). The academic achievement domain score was calculated from $\mathrm{Z}$-scores for the reading and mathematics measures. Neuropsychological assessments were divided into five domains: language (Boston Naming Test (29); Delis-Kaplan Executive Function Systems (DKEFS) Letter Fluency, Category Fluency, Color Naming, and Word Reading (30)) (i), visual-spatial perception (Benton Judgment of Line Orientation (31)) (ii); visual-motor integration (Bender Visual Motor Gestalt Test (32)) (iii), memory (Wechsler Intelligence Scale for Children-III Spatial Span (33); Color Span Test (34); Children's Memory Scale Dot Locations and Word Lists (35)) (iv), and executive function (Conners' Continuous Performance Test (36); DKEFS Category Switching, Inhibition, and Sorting (30)) (v). Domain scores for language, visual-spatial perception, visual-motor integration, memory, and executive function were calculated using $\mathrm{Z}$-scores of measures within the respective domains. For motor skills assessment, children completed the Grooved Pegboard Test for fine motor skills and the Physical and Neurological Examination for Soft Signs (PANESS) for measures of axial control, dysrhythmia, sequencing, repetitive movements, and overflow movements $(37,38)$. The motor skills domain score was calculated using $\mathrm{z}$-scores from the Grooved Pegboard Test and PANESS.

\section{Image Acquisition and Analysis}

Imaging was performed without sedation in a research-dedicated Siemens 3T TIM Trio scanner (Siemens Healthcare, Erlangen, Germany) using a 12-channel head coil. Prior to the scan, children completed a session in an MRI simulator to familiarize them with the experience. T1-weighted images were acquired using an MP-RAGE sequence $(\mathrm{TR}=2,300 \mathrm{~ms}, \mathrm{TE}=2.82 \mathrm{~ms}, \mathrm{TI}=1,100 \mathrm{~ms}$, slice flip angle $=10$ degrees, FOV $=180 \times 180 \times 224$, matrix $=256 \times 256 \times 240 \mathrm{~mm}$, and bandwidth $=200 \mathrm{~Hz} /$ pixel). Next, T2-weighted images were acquired using a $3 \mathrm{D}$ variable flip angle SPACE sequence $(\mathrm{TR}=9,910 \mathrm{~ms}$, $\mathrm{TE}=430 \mathrm{~ms}$, FOV $=180 \times 180 \times 192 \mathrm{~mm}$, and matrix $=256 \times 256 \times 128 \mathrm{~mm})$. Anatomical images were analyzed using the BRAINS software automated workup pipeline (39). This pipeline included spatial alignment, tissue classification, and automated labeling. Automated results were checked for validity by a trained technician. A discriminant tissue classification was performed, and a brain mask was created using an artificial neural network. Brain measures included ICV, which was separated into total tissue and total CSF volume. Total tissue then was separated into volumes for cerebrum (cerebral cortex, cerebral white matter), cerebellum, and brainstem. The cerebrum was further broken down into the four cerebral lobes, each with component gray and white matter volumes. Artificial neural networks were utilized to measure subcortical structures (40). In addition, cortical reconstruction and volumetric segmentation were performed with FreeSurfer software version 5.1, developed at the Martinos Center for Biomedical Imaging. Using FreeSurfer, cortical tissue volume, surface area, and thickness were calculated for the cortex as a whole and the four cerebral lobes.

\section{Statistical Analysis}

Statistical analysis performed with SPSS v22 (IBM Corporation, Armonk, NY). Analysis of covariance was performed for continuous measures, and Pearson Chi-square was used for categorical measures. All behavioral, cognitive, motor, and brain measures were analyzed with age and sex as covariates. To avoid a type II error, the behavioral, cognitive, and motor assessments were grouped into domains for analysis to minimize the number of assessments. Height was also treated as a covariate for ICV in order to control for overall body size. For all other brain measures, ratios were utilized to account for ICV. Data distribution was checked for normality using the Shapiro-Wilk test. When indicated, analyses were repeated using rank order for nonparametric data. Linear regression was performed to evaluate for age by group and sex by group interactions. An $\alpha$-level of 0.10 was used as the threshold to explore any age by group or sex by group interaction. An $\alpha$-level of 0.05 was used as the threshold for statistical significance for main effects.

\section{ACKNOWLEDGMENTS}

The authors acknowledge the children and their parents for their participation as well as Andrea M. Aerts and Christine Saenz for their facilitation of research visits.

\section{STATEMENT OF FINANCIAL SUPPORT}

The work was supported by the National Institutes of Health (5K12HD02774822, PI Raphael Hirsch, MD), Bethesda, MD, the Stead Family Department of Pediatrics, and the Division of Neonatology at the University of lowa.

Disclosure: The authors (Brumbaugh, Conrad, Lee, DeVolder, Zimmerman, Magnotta, Axelson, Nopoulos) declare no conflicts of interest.

\section{REFERENCES}

1. Engle WA, Tomashek KM, Wallman C; Committee on Fetus and Newborn, American Academy of Pediatrics. "Late-preterm" infants: a population at risk. Pediatrics 2007;120:1390-401.

2. Martin JA, Hamilton BE, Osterman MJK, Curtin SC, Mathews TJ. Births: final data for 2012. Natl Vital Stat Rep 2015;64:1-68.

3. Kinney HC. The near-term (late preterm) human brain and risk for periventricular leukomalacia: a review. Semin Perinatol 2006;30:81-8.

4. Morse SB, Zheng H, Tang Y, Roth J. Early school-age outcomes of late preterm infants. Pediatrics 2009;123:e622-9.

5. Lipkind HS, Slopen ME, Pfeiffer MR, McVeigh KH. School-age outcomes of late preterm infants in New York City. Am J Obstet Gynecol 2012;206:222.e1-6.

6. Chan E, Quigley MA. School performance at age 7 years in late preterm and early term birth: a cohort study. Arch Dis Child Fetal Neonatal Ed 2014;99:F451-7.

7. de Kieviet JF, Zoetebier L, van Elburg RM, Vermeulen RJ, Oosterlaan J. Brain development of very preterm and very low-birthweight children in childhood and adolescence: a meta-analysis. Dev Med Child Neurol 2012;54:313-23.

8. Lax ID, Duerden EG, Lin SY, et al. Neuroanatomical consequences of very preterm birth in middle childhood. Brain Struct Funct 2013;218:575-85.

9. Munakata S, Okada T, Okahashi A, et al. Gray matter volumetric MRI differences late-preterm and term infants. Brain Dev 2013;35:10-6.

10. Walsh JM, Doyle LW, Anderson PJ, Lee KJ, Cheong JL. Moderate and late preterm birth: effect on brain size and maturation at term-equivalent age. Radiology 2014;273:232-40.

11. Rogers CE, Barch DM, Sylvester CM, et al. Altered gray matter volume and school age anxiety in children born late preterm. J Pediatr 2014;165: 928-35.

12. Lenroot RK, Gogtay N, Greenstein DK, et al. Sexual dimorphism of brain developmental trajectories during childhood and adolescence. Neuroimage 2007;36:1065-73.

13. Sowell ER, Thompson PM, Tessner KD, Toga AW. Mapping continued brain growth and gray matter density reduction in dorsal frontal cortex: Inverse relationships during postadolescent brain maturation. J Neurosci 2001;21:8819-29.

14. Shaw P, Greenstein D, Lerch J, et al. Intellectual ability and cortical development in children and adolescents. Nature 2006;440:676-9.

15. Ment LR, Kesler S, Vohr B, et al. Longitudinal brain volume changes in preterm and term control subjects during late childhood and adolescence. Pediatrics 2009;123:503-11.

16. Nagasaka M, Morioka I, Yokota T, et al. Incidence of short stature at 3 years of age in late preterm infants: a population-based study. Arch Dis Child 2015;100:250-4.

17. Ramel SE, Demerath EW, Gray HL, Younge N, Boys C, Georgieff MK. The relationship of poor linear growth velocity with neonatal illness and twoyear neurodevelopment in preterm infants. Neonatology 2012;102:19-24.

18. Lindström K, Lindblad F, Hjern A. Preterm birth and attention-deficit/ hyperactivity disorder in schoolchildren. Pediatrics 2011;127:858-65.

19. Mulder H, Pitchford NJ, Marlow N. Processing speed and working memory underlie academic attainment in very preterm children. Arch Dis Child Fetal Neonatal Ed 2010;95:F267-72.

20. Clark CA, Woodward LJ. Neonatal cerebral abnormalities and later verbal and visuospatial working memory abilities of children born very preterm. Dev Neuropsychol 2010;35:622-42. 
21. Baron IS, Erickson K, Ahronovich MD, Coulehan K, Baker R, Litman FR. Visuospatial and verbal fluency relative deficits in 'complicated' late-preterm preschool children. Early Hum Dev 2009;85:751-4.

22. Nosarti C, Giouroukou E, Healy E, et al. Grey and white matter distribution in very preterm adolescents mediates neurodevelopmental outcome. Brain 2008;131(Pt 1):205-17.

23. Taylor HG, Filipek PA, Juranek J, Bangert B, Minich N, Hack M. Brain volumes in adolescents with very low birth weight: effects on brain structure and associations with neuropsychological outcomes. Dev Neuropsychol 2011;36:96-117.

24. Parker S, Greer S, Zuckerman B. Double jeopardy: the impact of poverty on early child development. Pediatr Clin North Am 1988;35:122740.

25. Hollingshead AA. Four-factor index of social status. In: Smith P, ed. Yale Journal of Sociology. Vol 8. New Haven, CT: Yale University, 2011:21-51.

26. Lindgren SD, Koeppl GK. Assessing child behavior problems in a medical setting: Development of the Pediatric Behavior Scale. In: Prinz RJ, ed. Advances in behavioral assessment of children and families. Greenwich, CT: JAI Press, Inc., 1987:57-90.

27. Wechsler D, Kaplan E, Fein D, et al. Wechsler Intelligence Scale for Children Integrated: Administration and Scoring Manual. 4th edn. San Antonio, TX: Harcourt Assessment, Inc., 2004.

28. Wilkinson GS, Robertson GJ. Wide Range Achievement Test. 4th edn. Lutz, FL: Psychological Assessment Resources, Inc., 2006.

29. Kaplan E, Goodglass H, Weintraub S. Boston Naming Test. Philadelphia, PA: Lea \& Febiger, 1983.
30. Delis DC, Kaplan E, Kramer JH. Delis-Kaplan Executive Function System. San Antonio, TX: The Psychological Corporation, 2001.

31. Benton AL, Hamsher K, Sivan AB. Multilingual Aphasia Examination. 3rd edn. Iowa City, IA: AJA Associates, 1994.

32. Brannigan GG, Decker SL. Bender Visual-Motor Gestalt Test. 2nd edn. Itasca, IL: Riverside Publishing, 2003.

33. Wechsler D. Wechsler Intelligence Scale for Children Manual. 3rd edn. Washington, D.C.: The Psychological Corporation, 1991.

34. Richman LC, Lindgren SD. The Color Span Test. Iowa City, IA: University of Iowa, 1978.

35. Cohen MJ. Children's Memory Scale. San Antonio, TX: The Psychological Corporation, 1997.

36. Conners CK. Conners' Continuous Performance Test II: Computer Program for Windows Technical Guide and Software Manual. North Tonawanda, NY: Multi-Helath Systems, 2000.

37. Grooved Pegboard Test User Instructions. Lafayette, IN: Lafayette Instrument, 2002. (http://www.si-instruments.com.au/industry/download/lafayette-current-version-grooved-pegboard-test-32025-lafayette32025-grooved-pegboard-test-manual-pdf.html)

38. Denckla MB. Revised neurological examination for subtle signs (1985). Psychopharmacol Bull 1985;21:773-800.

39. Pierson R, Johnson H, Harris G, et al. Fully automated analysis using BRAINS: AutoWorkup. Neuroimage 2011;54:328-36.

40. Powell S, Magnotta VA, Johnson H, Jammalamadaka VK, Pierson R, Andreasen NC. Registration and machine learning-based automated segmentation of subcortical and cerebellar brain structures. Neuroimage 2008;39:238-47. 